PROCEEDINGS OF THE

AMERICAN MATHEMATICAL SOCIETY

Volume 126, Number 3, March 1998, Pages 881-885

S $0002-9939(98) 04420-7$

\title{
FIXED POINTS OF THE BUCKET HANDLE
}

\author{
JAN M. AARTS AND ROBBERT J. FOKKINK
}

(Communicated by James West)

\begin{abstract}
If a homeomorphism on the bucket handle has an invariant composant, it has a fixed point in that composant. It follows that a homeomorphism on the bucket handle has at least two fixed points. Our methods apply to general Knaster continua.
\end{abstract}

\section{INTRODUCTION}

Mahavier has asked whether every homeomorphism of the standard Knaster continuum or bucket handle has at least two fixed points [3, p 384, Problem 120]. In this paper we give a positive answer to this question. Our proof makes use of the fact that the 2 -solenoid is a branched covering space of the bucket handle. The structure of the isotopy class group of the solenoid is also involved in the proof. See Section 2 for details. The proof is presented in Section 3. In the remainder of this section we recall some notation.

The solenoid and bucket handle will be represented as limits of inverse sequences. We consider inverse sequences

$$
\text { .. } \stackrel{f}{\longrightarrow} X \stackrel{f}{\longrightarrow} X \stackrel{f}{\longrightarrow} X .
$$

The shift $\sigma$ of the inverse limit is defined by $\sigma\left(\left\langle x_{n}\right\rangle_{n}\right)=\left\langle x_{n+1}\right\rangle_{n}$.

The bucket handle is the inverse limit of the sequence (1) with $X=I$, the unit interval, and $f=\tau$, the tent map which is defined by $\tau(x)=\min \{2 x, 2-2 x\}$. The bucket handle is denoted by $\mathcal{K}$ and the shift on $\mathcal{K}$ is denoted by $\sigma_{\mathcal{K}}$. Since the tent map has two fixed points, so does the shift $\sigma_{\mathcal{K}}$. The sequence $\langle 0\rangle_{n}$ in $\mathcal{K}$ is called the initial point and is denoted by $0_{\mathcal{K}}$. The initial point is topologically unique. Thus the initial point is a fixed point of every homeomorphism of the bucket handle. Mahavier's question is: does there exist yet another fixed point?

The circle $\mathbb{R} / \mathbb{Z}$ is denoted by $C$. Via the quotient map $C$ is identified with $[0,1)$. The circle $C$ has the structure of an additive topological group; the sum of $x$ and $y$ is $x+y(\bmod 1)$. The 2-solenoid, or just solenoid, is the inverse limit of the sequence (1) with $X=C$ and $f=\delta$, the doubling map which is defined by $\delta(x)=2 x$ $(\bmod 1)$. The solenoid is denoted by $\mathcal{S}$ and the shift on $\mathcal{S}$ is denoted by $\sigma_{\mathcal{S}}$. The solenoid is a compact abelian group. The group operation is coordinatewise addition. The zero element is $\langle 0\rangle_{n}$. It is denoted by $0_{\mathcal{S}}$.

Received by the editors December 15, 1995.

1991 Mathematics Subject Classification. Primary 54H25, 54F15; Secondary 54H11.

Key words and phrases. Fixed point, bucket handle, solenoid.

(C) 1998 American Mathematical Society 
Note that the restriction of the tent map to $[0,1)$ maps $C$ onto $I$. Thus $\tau$ is a semi-conjugacy of $\delta$ to $\tau: \tau \circ \delta=\tau \circ \tau$. It follows that $\tau$ induces a semi-conjugacy, denoted by $\pi$, of $\sigma_{\mathcal{S}}$ to $\sigma_{\mathcal{K}}$, namely, $\pi\left(\left\langle x_{n}\right\rangle_{n}\right)=\left\langle\tau\left(x_{n}\right)\right\rangle_{n}$. We have $\pi \circ \sigma_{\mathcal{S}}=\sigma_{\mathcal{K}} \circ \pi$. Note that $\pi\left(0_{\mathcal{S}}\right)=0_{\mathcal{K}}$. Hence $\pi$ is a map of the pointed space $\left(\mathcal{S}, 0_{\mathcal{S}}\right)$ to the pointed space $\left(\mathcal{K}, 0_{\mathcal{K}}\right)$. The following result is well known.

Proposition 1. Let $\rho: \mathcal{S} \rightarrow \mathcal{S}$ be the involution defined by $\rho(x)=-x$. Then $\pi=\pi \circ \rho$. For all $x, y \in \mathcal{S}, \pi(x)=\pi(y)$ if and only if $x=y$ or $x=\rho(y)$.

The relation between the bucket handle and the solenoid is very much that between a non-orientable manifold and its orientable cover. In this sense the involution $\rho$ is a deck transformation. As is stated in the next theorem, the projection map $\pi: \mathcal{S} \rightarrow \mathcal{K}$ has the isotopy lifting property [1]. Recall that homeomorphisms $g, h:(X, p) \rightarrow(X, p)$ of the pointed space $(X, p)$ are isotopic, $g \sim h$, if there exists a continuous map $H: X \times I \rightarrow X$ such that $H(\cdot, t): X \rightarrow X$ is a homeomorphism with $H(p, t)=p$ for all $t \in I, H(\cdot, 0)=g$ and $H(\cdot, 1)=h$. We observe that the composants of $\mathcal{S}$ and $\mathcal{K}$ are just the arc components.

Theorem 2. Let $H: \mathcal{K} \times I \rightarrow \mathcal{K}$ be an isotopy on the bucket handle. There exists an isotopy $\widetilde{H}: \mathcal{S} \times I \rightarrow \mathcal{S}$ such that $H \circ \pi=\pi \circ \widetilde{H}$. The isotopy lifting property also holds for the pointed spaces $\left(\mathcal{K}, 0_{\mathcal{K}}\right)$ and $\left(\mathcal{S}, 0_{\mathcal{S}}\right)$.

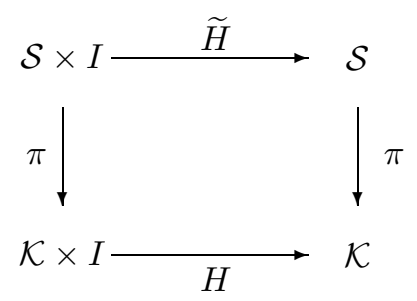

\section{LifTing HOMEOMORPHISMS OF THE BUCKET HANDLE}

We discuss the relation between homeomorphisms on the solenoid and homeomorphisms on the bucket handle. The reader is referred to [1] for details.

It is well known that the solenoid admits a flow without rest points. Here is an explicit construction of such a flow $\varphi$. The sequence $s(t)=\left\langle t / 2^{n}(\bmod 1)\right\rangle_{n}$ is an element of the solenoid for each $t \in \mathbb{R}$. Note that $s$ is a continuous group isomorphism of $\mathbb{R}$ to the dense one-parameter subgroup $\Gamma=s(\mathbb{R})$ of $\mathcal{S}$. The flow $\varphi: \mathcal{S} \times \mathbb{R} \rightarrow \mathcal{S}$ is defined by $\varphi(x, t)=x+s(t)$. We make the following observations.

1. The one-parameter subgroup $\Gamma$ coincides with the composant of $0_{\mathcal{S}}$.

2. The orbits of the flow coincide with the composants of the solenoid. The composant of the point $p$ equals $p+\Gamma$. It is denoted by $\Gamma(p)$.

3. The restriction $\rho: \Gamma \rightarrow \Gamma$ is given by $x(t) \rightarrow x(-t)$.

4. The restriction $\sigma_{\mathcal{S}}: \Gamma \rightarrow \Gamma$ is given by $x(t) \rightarrow x\left(\frac{t}{2}\right)$.

Lemma 3. Let $h: \mathcal{K} \rightarrow \mathcal{K}$ be a homeomorphism. There are exactly two homeomorphisms $\widetilde{h}_{1}, \widetilde{h}_{2}: \mathcal{S} \rightarrow \mathcal{S}$ of the solenoid that are lifts of $h$, i.e., $\pi \circ \widetilde{h}_{i}=h \circ \pi$ $(i=1,2)$. Furthermore, $\rho \circ \widetilde{h}_{i}=\widetilde{h}_{j}$ for $i \neq j$.

Proof. According to Theorem 2 the homeomorphism $h$ can be lifted to a homeomorphism $\widetilde{h}$ of the solenoid. Note that $\widetilde{h} \circ \rho$ is another lift. We have to show that there 
are no other lifts. First consider the special case that $h$ equals the identity $i d_{\mathcal{K}}$. Let $\widetilde{h}: \mathcal{S} \rightarrow \mathcal{S}$ be a lift of the identity. Then by Proposition 1

$$
\mathcal{S}=\{x \mid \widetilde{h}(x)=x\} \cup\{x \mid \widetilde{h}(x)=\rho(x)\},
$$

the union of two closed sets with intersection $\left\{0_{\mathcal{S}}\right\}$. Since $\mathcal{S} \backslash\left\{0_{\mathcal{S}}\right\}$ is connected, one of these sets must be empty. It follows that either $\widetilde{h}=i d_{\mathcal{S}}$ or $\widetilde{h}=\rho$. Hence the statement is true in this case. Now consider the general case. Let $\widetilde{h}_{1}$ and $\widetilde{h}_{2}$ be lifts of $h$. If $\widetilde{g}$ is a lift of the inverse of $h$, then both $\widetilde{h}_{1} \circ \widetilde{g}$ and $\widetilde{h}_{2} \circ \widetilde{g}$ are lifts of the identity. The result now follows easily.

Corollary 4. Suppose that $\widetilde{h}: \mathcal{S} \rightarrow \mathcal{S}$ is a lift of the homeomorphism $h: \mathcal{K} \rightarrow \mathcal{K}$. Then $\widetilde{h}$ commutes with $\rho$.

We now collect some results from [1] concerning isotopy classes of homeomorphisms of $\mathcal{S}$ and $\mathcal{K}$. The isotopy class of a homeomorphism $h$ is denoted by $[h]$. The class group $\mathcal{C}(X, p)$ is the group of isotopy classes of base point preserving homeomorphisms. The group of automorphisms of a topological group $G$ is denoted by $\operatorname{Aut}(G)$. The proof of the following theorem goes back to [4].

Theorem 5. The map $i: A u t(\mathcal{S}) \rightarrow \mathcal{C}\left(\mathcal{S}, 0_{\mathcal{S}}\right)$ defined by $i(h)=[h]$ is an isomorphism.

Theorem 6. $\operatorname{Aut}(\mathcal{S}) \simeq \mathbb{Z} \oplus \mathbb{Z} / 2 \mathbb{Z}$

Corollary 7. The subgroup of orientation-preserving isotopy classes in $\mathcal{C}\left(\mathcal{S}, 0_{\mathcal{S}}\right)$ is infinite cyclic and generated by $\sigma_{\mathcal{S}}$.

Theorem 8. The class group $\mathcal{C}\left(\mathcal{K}, 0_{\mathcal{K}}\right)$ is infinite cyclic. It is generated by $\sigma_{\mathcal{K}}$.

\section{Fixed POINTS ON THE BUCKET HANDLE}

We say that a homeomorphism $h$ has an invariant set $A$ if $h(A) \subset A$. We first prove that each homeomorphism of the bucket handle has at least two invariant composants. Then we show that if a homeomorphism of the bucket handle has an invariant composant, it has a fixed point in that composant.

Lemma 9. A homeomorphism on the bucket handle has at least two invariant composants.

Proof. Each homeomorphism $h$ on the bucket handle is isotopic to an iterate of $\sigma_{\mathcal{K}}$. Note that if $H$ is an isotopy then $H(x, t)$ and $H(x, s)$ belong to the same composant for all $s, t \in I$. It therefore suffices to show that $\sigma_{\mathcal{K}}$ has two invariant composants. The points $0_{\mathcal{K}}$ and $\left\langle\frac{2}{3}\right\rangle_{n}$ are fixed points of $\sigma_{\mathcal{K}}$. We complete the proof by showing that these fixed points are not contained in the same composant. The preimage of $0_{\mathcal{K}}$ in the solenoid is $0_{\mathcal{S}}$. Now

$$
\pi^{-1}\left(\left\langle\frac{2}{3}\right\rangle_{n}\right)=\left\{\left\langle\frac{(-1)^{n}}{3} \quad(\bmod 1)\right\rangle_{n},\left\langle\frac{(-1)^{n+1}}{3} \quad(\bmod 1)\right\rangle_{n}\right\} .
$$

It follows that $\pi^{-1}\left(\langle 2 / 3\rangle_{n}\right)$ and the composant of $0_{\mathcal{S}}$ are disjoint. Hence the fixed points $0_{\mathcal{K}}$ and $\left\langle\frac{2}{3}\right\rangle_{n}$ are contained in different composants.

Now we show that if a homeomorphism of $\mathcal{K}$ has an invariant composant, it has a fixed point in that composant. This requires some preliminary work. The following lemma is a direct consequence of [2, Theorem 1.25]. 
Lemma 10. Let $G$ be a compact and connected subset of $\Gamma$. Then there is a compact interval $J$ in $\mathbb{R}$ such that $s(J)=G$.

Note that $s: \mathbb{R} \rightarrow \Gamma$ is an isomorphism of groups.

Lemma 11. Let $\alpha: \mathcal{S} \rightarrow \mathcal{S}$ be a continuous homomorphism. Then either $\alpha(\Gamma)=\Gamma$ or $\alpha(\Gamma)=\left\{0_{\mathcal{S}}\right\}$.

Proof. As $\alpha$ is a homomorphism, $0_{\mathcal{S}} \in \Gamma \cap \alpha(\Gamma)$. It follows that $\alpha(\Gamma) \subset \Gamma$. Define $\widetilde{\alpha}=s^{-1} \circ \alpha \circ s$. By Lemma 10, $\widetilde{\alpha}$ is continuous on each compact interval, hence continuous. Because $\widetilde{\alpha}$ is also a homomorphism, $\widetilde{\alpha}(\mathbb{R})$ is a connected subgroup of $\mathbb{R}$. The lemma follows.

Recall that the composant of a point $p$ of $\mathcal{S}$ is $\Gamma(p)=p+\Gamma$.

Lemma 12. Let $p \in \mathcal{S}$. Suppose that $g: \mathcal{S} \rightarrow \mathcal{S}$ is a homeomorphism such that $g(\Gamma(p)) \subset \Gamma(p)$. Suppose $g(x) \neq x$ for all $x \in \Gamma(p)$. Then $\left(g-i d_{\mathcal{S}}\right)(\Gamma(p))$ is contained in a compact subset of $\Gamma$.

Proof. By Theorem 5, $g$ is isotopic to an automorphism $\gamma: \mathcal{S} \rightarrow \mathcal{S}$. Note that $g(x)$ and $\gamma(x)$ belong to the same composant for each $x \in \mathcal{S}$. Consequently $g(x)-$ $\gamma(x) \in \Gamma$ for all $x$. Define $\beta=g-\gamma$ and $\alpha=\gamma-\mathrm{id}_{\mathcal{S}}$. As the cosets of $\Gamma$ coincide with the composants, $\beta(\mathcal{S})$ is a compact subset of $\Gamma$. Note that $\alpha$ is a continuous homomorphism. In view of Lemma 11, there are two cases: $\alpha(\Gamma)=\Gamma$ or $\alpha(\Gamma)=\left\{0_{\mathcal{S}}\right\}$. In the latter case $\alpha(\mathcal{S})=0_{\mathcal{S}}$, whence $\gamma$ equals id $\mathcal{S}_{\mathcal{S}}$ and $\beta=g-\mathrm{id}_{\mathcal{S}}$. In particular, $g-\operatorname{id}_{\mathcal{S}}(\Gamma(p))=\beta(\Gamma(p)) \subset \beta(\mathcal{S})$, which is a compact subset of $\Gamma$, and the proof is complete. So we may assume that $\alpha(\Gamma)=\Gamma$. Under this assumption by an intermediate value argument we shall show that there exists a $z \in \Gamma$ such that $g(z+p)=z+p$. This is impossible, as $g(x) \neq x$ for all $x \in \Gamma(p)$.

As $g(\Gamma(p)) \subset \Gamma(p)$, we have $g(p+y)-(p+y) \in \Gamma$ for all $y \in \Gamma$. It follows that for all $y \in \Gamma$

$$
\begin{aligned}
g(p+y)-(p+y) & =\gamma(p+y)-(p+y)+\beta(p+y) \\
& =\alpha(p)+\alpha(y)+\beta(p+y) .
\end{aligned}
$$

The intermediate value argument follows. As $\beta$ is continuous, $\beta(\mathcal{S})$ is a compact and connected subset of $\Gamma$. Note that $\alpha$ and $\beta(p+\cdot)$ send $\Gamma$ to $\Gamma$. As in the proof of Lemma 11 there are continuous lifts $\widetilde{\alpha}: \mathbb{R} \rightarrow \mathbb{R}$ and $\widetilde{\beta^{\prime}}: \mathbb{R} \rightarrow \mathbb{R}$ of $\alpha$ and $\beta(p+\cdot)$ respectively. As $\widetilde{\alpha}(\mathbb{R})=\mathbb{R}$ and $\widetilde{\beta^{\prime}}(\mathbb{R})$ is compact, $\widetilde{\alpha}+\widetilde{\beta}^{\prime}$ maps $\mathbb{R}$ onto itself. It follows that for some $x$ in $\mathbb{R}$ we have, writing $s(x)=z$,

$$
s \circ\left(\widetilde{\alpha}+\widetilde{\beta}^{\prime}\right)(x)=\alpha(z)+\beta(p+z)=-\alpha(p) .
$$

It follows that $\left(g-i d_{\mathcal{S}}\right)(p+z)=0_{\mathcal{S}}$.

Lemma 13. If a homeomorphism on the bucket handle has an invariant composant, it has a fixed point in that composant.

Proof. Suppose that $g: \mathcal{K} \rightarrow \mathcal{K}$ has an invariant composant $\Gamma(p)$. We may assume that $\Gamma(p)$ is not the composant of the initial point, since the initial point is a fixed point of every homeomorphism of $\mathcal{K}$. The preimage of $\Gamma(p)$ in $\mathcal{S}$ under $\pi$ is the union of two composants $\Gamma_{1}, \Gamma_{2}$ of $\mathcal{S}$, which are interchanged by the involution $\rho$. Let $\widetilde{g}$ be the lift of $g$ which has both $\Gamma_{1}$ and $\Gamma_{2}$ as an invariant set. We show that $\widetilde{g}$ has a fixed point on $\Gamma_{1} \cup \Gamma_{2}$. The proof is by contradiction. If there is no fixed point, then by Lemma 12 the map $\widetilde{h}=\widetilde{g}-i d_{\mathcal{S}}$ sends $\Gamma_{1}$ into a compact subset of $\Gamma$. The 
same holds true for $\Gamma_{2}$. Now observe that $\widetilde{h} \circ \rho=\rho \circ \widetilde{h}$, so $A=\widetilde{h}\left(\Gamma_{1} \cup \Gamma_{2}\right)$ is invariant under $\rho$. Note that $A$ is contained in a compact subset $K$ of $\Gamma$. Furthermore, $A$ is connected, since $\Gamma_{1} \cup \Gamma_{2}$ is connected. So we may assume that $K$ is connected as well. As in the proof of the previous lemma, there is a compact interval $J$ in $\mathbb{R}$ such that $s(J)=K$. The restriction of $s^{-1}$ to $K$ is a homeomorphism. It follows that $s^{-1}(A)$ is a connectected subset of $\mathbb{R}$ such that $s^{-1}(A)=-s^{-1}(A)$. Consequently $0 \in s^{-1}(A)$. Hence $0_{\mathcal{S}} \in A$, so $\widetilde{h}$ has a fixed point in $\Gamma_{1} \cup \Gamma_{2}$.

Lemma 9 and Lemma 13 imply our main result.

Theorem 14. A homeomorphism on the bucket handle has at least two fixed points.

This result can be extended as follows. For each $m$ in $\mathbb{N}$ we define the tentlike map $\tau_{m}: I \rightarrow I$ by periodic extension of the map $\tau_{m}(x)=\min \{m x, 2-m x\}$, $x \in\left[0, \frac{2}{m}\right]$. The inverse limit of the sequence (1) with $X=I$ and $f=\tau_{m}$ is the Knaster continuum $\mathcal{K}_{m}$. In this terminology the bucket handle is equal to $\mathcal{K}_{2}$.

Theorem 15. Let $p \in \mathbb{N}$ be a prime number. Let $g: \mathcal{K}_{p} \rightarrow \mathcal{K}_{p}$ be a homeomorphism on the Knaster continuum $\mathcal{K}_{p}$. The number of points in $\left\{x \mid g^{n}(x)=x\right\}$ is at least equal to $p \cdot n$.

The proof of this theorem involves a straightforward generalization of our proof of Theorem 14. The method used in this paper, in particular Lemma 12, remains valid for even the most general Knaster continua.

\section{REFERENCES}

[1] J. M. Aarts and R. J. Fokkink, On composants of the bucket handle, Fundamenta Mathematicae 139 (1991), 194-208. MR 93a:54030

[2] A. Beck, Continuous flows in the plane, Springer, Berlin 1974. MR 58:18379

[3] H. Cook, W. T. Ingram and A. Lelek, A list of problems known as Houston Problem Book, Continua with the Houston problem book, H. Cook, W. T. Ingram, K. T. Kuperberg, A. Lelek and P. Minc (eds), Lecture Notes in pure and applied mathematics 170, Marcel Dekker, New York (N.Y.) 1995, 365-398. MR 96f:54042

[4] D. van Dantzig, Ueber topologisch homogene Kontinua, Fundamenta Mathematicae 14 (1930), 102-125.

Technical University Delft, Faculty of Mathematics and Informatics, P.O. Box 5031, 2600 GA Delft, the Netherlands

E-mail address: j.m.aarts@twi.tudelft.nl

Delft Hydraulics, Rotterdamseweg 181, 2600 MH Delft, the Netherlands

E-mail address: fokkink@nsld.wldelft.nl 